\title{
Pacific
}

Journal of

Mathematics

\section{ON OCCULT PERIOD MAPS}

Stephen Kudla And Michael Rapoport 


\title{
ON OCCULT PERIOD MAPS
}

\author{
STEPHEN KUdLA AND Michael RAPOPORT
}

In memoriam Jonathan Rogawski

\begin{abstract}
We interpret the "occult" period maps of Allcock, Carlson, and Toledo (2002; 2011), of Looijenga and Swierstra (2007; 2008), and of Kondō (2000; 2002) in moduli theoretic terms, as a construction of certain families of polarized abelian varieties of Picard type. We show that these period maps are morphisms defined over their natural field of definition.
\end{abstract}

\section{Introduction}

In papers of Allcock, Carlson, and Toledo [Allcock et al. 2002; 2011], of Looijenga and Swierstra [2007; 2008], and of Kondō [2000; 2002], "hidden" period maps are constructed in certain cases. The target spaces of these maps are certain arithmetic quotients of complex unit balls. The basic observation which is the starting point of this paper is that these arithmetic quotients can be interpreted as the complex points of certain moduli spaces of abelian varieties of Picard type, of the kind considered in [Kudla and Rapoport 2009]. Consequently, the purpose in this paper is to interpret these hidden period maps in moduli-theoretic terms. The payoff of this exercise is that we can raise and partially answer some descent problems which seem natural from our viewpoint, and which are related to a similar descent problem addressed by Deligne [1972] in his theory of complete intersections of Hodge level one.

Why do we speak of "hidden" or "occult" period maps in this context? This is done in order to make the distinction with the usual period maps which associate to a family of smooth projective complex varieties (over some base scheme $S$ ) the (polarized) Hodge structures of its fibers, which then induces a map from $S$ to a quotient by a discrete group of a period domain. Let us recall three examples of classical period maps:

(1) Case of quartic surfaces. In this case, the period map is a holomorphic map of orbifolds

$$
\varphi: \text { Quartics }{ }_{2, \mathbb{C}}^{\circ} \rightarrow[\Gamma \backslash V(2,19)] .
$$

MSC2010: 11G15, 14D20, 14K22.

Keywords: Torelli theorems, period maps. 
Here Quartics ${ }_{2, \mathbb{C}}^{\circ}$ denotes the stack parametrizing smooth quartic surfaces up to projective equivalence,

$$
\text { Quartics }_{2, \mathbb{C}}^{\circ}=\left[\mathrm{PGL}_{4} \backslash \operatorname{PSym}^{4}\left(\mathbb{C}^{4}\right)^{\circ}\right]
$$

(stack quotient in the orbifold sense). The target space is the orbifold quotient of the space of oriented positive 2-planes in a quadratic space $V$ of signature $(2,19)$ by the automorphism group $\Gamma$ of a lattice in $V$.

(2) Case of cubic threefolds. In this case, the period map is a holomorphic map of orbifolds

$$
\varphi: \operatorname{Cubics}_{3, \mathbb{C}}^{\circ} \rightarrow\left[\Gamma \backslash \mathfrak{H}_{5}\right] .
$$

Here Cubics ${ }_{3, \mathbb{C}}^{\circ}$ denotes the stack parametrizing smooth cubic threefolds up to projective equivalence. The target space is the orbifold quotient of the Siegel upper half-space of genus 5 by the Siegel group $\Gamma=\mathrm{Sp}_{5}(\mathbb{Z})$.

(3) Case of cubic fourfolds. In this case, the period map is a holomorphic map of orbifolds

$$
\varphi: \text { Cubics }_{4, \mathbb{C}}^{\circ} \rightarrow[\Gamma \backslash V(2,20)] .
$$

Here Cubics ${ }_{4, \mathbb{C}}^{\circ}$ denotes the stack parametrizing smooth cubic fourfolds up to projective equivalence. The target space is the orbifold quotient of the space of oriented positive 2-planes in a quadratic space $V$ of signature $(2,20)$ by the automorphism group $\Gamma$ of a lattice in $V$.

In the first case, by the Torelli theorem of Piatetski-Shapiro and Shafarevich, the induced map $|\varphi|$ on coarse moduli spaces is an open embedding. In the second case, by the Torelli theorem of Clemens and Griffiths, the map $|\varphi|$ is a locally closed embedding (it is not an open embedding since the source of $\varphi$ has dimension 10, and the target has dimension 15). In the third case, by the Torelli theorem of Voisin, the map $|\varphi|$ is an open embedding.

The construction of the occult period maps is quite different, although it does use the classical period maps indirectly. For instance, the construction of Allcock, Carlson, and Toledo attaches a certain Hodge structure to any smooth cubic surface which allows one to distinguish between nonisomorphic ones, even though the natural Hodge structures on the cohomology in the middle dimension of all cubic surfaces are isomorphic. Also, in one dimension higher, their construction allows them to define an open embedding of the space of cubic threefolds into an arithmetic quotient of the complex unit ball of dimension 10.

Our second aim in this paper is to identify the complements of the images of occult period maps with special divisors considered in [Kudla and Rapoport 2009].

The layout of the paper is as follows. In Sections 2, 3 and 4, we recall some of the theory and notation of [Kudla and Rapoport 2009]. In Sections 5, 6, 7 and 8, 
respectively, we explain in turn the cases of cubic surfaces, cubic threefolds, curves of genus 3, and curves of genus 4. In Section 9, we explain the descent problem and solve it in zero characteristic. In the final section, we make a few supplementary remarks.

We stress that the proofs of our statements are all contained in the papers mentioned above, and that our work only consists in interpreting these results.

\section{Moduli spaces of Picard type}

Let $\boldsymbol{k}=\mathbb{Q}(\sqrt{\Delta})$ be an imaginary-quadratic field with discriminant $\Delta$, ring of integers $O_{k}$, and a fixed complex embedding. We write $a \mapsto a^{\sigma}$ for the nontrivial automorphism of $O_{\boldsymbol{k}}$.

For integers $n \geq 1$ and $r, 0 \leq r \leq n$, we consider the groupoid $\mathcal{M}=\mathcal{M}(n-r, r)=$ $\mathcal{M}(\boldsymbol{k} ; n-r, r)$ fibered over $\left(\mathrm{Sch} / O_{k}\right)$ which associates to an $O_{\boldsymbol{k}}$-scheme $S$ the groupoid of triples $(A, \iota, \lambda)$. Here $A$ is an abelian scheme over $S, \lambda$ is a principal polarization, and $\iota: O_{k} \rightarrow \operatorname{End}(A)$ is a homomorphism such that

$$
\iota(a)^{*}=\iota\left(a^{\sigma}\right),
$$

for the Rosati involution $*$ corresponding to $\lambda$. In addition, the following signature condition is imposed:

$$
\operatorname{char}(T, \iota(a) \mid \operatorname{Lie} A)=(T-i(a))^{n-r} \cdot\left(T-i\left(a^{\sigma}\right)\right)^{r}, \quad \text { for all } a \in O_{k},
$$

where $i: O_{k} \rightarrow O_{S}$ is the structure map.

We will mostly consider the complex fiber $M_{\mathbb{C}}=M \times_{\operatorname{Spec} O_{k}} \operatorname{Spec} \mathbb{C}$ of $M$. In any case, $\mathcal{M}$ is a Deligne-Mumford stack and $\mathcal{M}_{\mathbb{C}}$ is smooth. We denote by $\left|\mathcal{M}_{\mathbb{C}}\right|$ the coarse moduli scheme.

We will also have to consider the following variant, defined by modifying the requirement above that the polarization $\lambda$ be principal. Let $d>1$ be a squarefree divisor of $|\Delta|$. Then $\mathcal{M}(\boldsymbol{k}, d ; n-r, r)^{*}=\mathcal{M}(\boldsymbol{k} ; n-r, r)^{*}$ parametrizes triples $(A, \iota, \lambda)$ as in the case of $M(k ; n-r, r)$, except that we impose the following condition on $\lambda$. We require first of all that $\operatorname{ker} \lambda \subset A[d]$, so that $O_{k} /(d)$ acts on $\operatorname{ker} \lambda$. In addition, we require that this action factor through the quotient ring $\prod_{p \mid d} \mathbb{F}_{p}$ of $O_{k} /(d)$, and that $\lambda$ be of degree $d^{n-1}$ if $n$ is odd and of degree $d^{n-2}$ if $n$ is even. In the notation introduced in Section 13 of [Kudla and Rapoport 2009], we have $\mathcal{M}(\boldsymbol{k}, d ; n-r, r)^{*}=\mathcal{M}(\boldsymbol{k}, \mathbf{t} ; n-r, r)^{* \text {,naive }}$, where the function $\mathbf{t}$ on the set of primes $p$ with $p \mid \Delta$ assigns to $p$ the integer 2[(n-1)/2] if $p \mid d$, and 0 if $p \nmid d$. Note that if $\boldsymbol{k}$ is the Gaussian field $\boldsymbol{k}=\mathbb{Q}(\sqrt{-1})$, then necessarily $d=2$; if $\boldsymbol{k}$ is the Eisenstein field $\boldsymbol{k}=\mathbb{Q}(\sqrt{-3})$, then $d=3$. We denote by $\left|M_{\mathbb{C}}^{*}\right|$ the corresponding coarse moduli scheme. 


\section{Complex uniformization}

Let us recall from [Kudla and Rapoport 2009] the complex uniformization of $\mathcal{M}(\boldsymbol{k} ; n-1,1)(\mathbb{C})$ in the special case that $\boldsymbol{k}$ has class number one. For $n>2$, let $(V,()$,$) be a hermitian vector space over \boldsymbol{k}$ of signature $(n-1,1)$ which contains a self-dual $O_{k}$-lattice $L$. By the class number hypothesis, $V$ is unique up to isomorphism. When $n$ is odd, or when $n$ is even and $\Delta$ is odd, the lattice $L$ is also unique up to isomorphism. We assume that one of these conditions is satisfied. Let $\mathscr{D}$ be the space of negative lines in the $\mathbb{C}$-vector space $\left(V_{\mathbb{R}}, \mathbb{\square}_{0}\right)$, where the complex structure $\rrbracket_{0}$ is defined in terms of the discriminant of $\boldsymbol{k}$, as $\rrbracket_{0}=\sqrt{\Delta} /|\sqrt{\Delta}|$. Let $\Gamma$ be the isometry group of $L$. Then the complex uniformization is the isomorphism of orbifolds,

$$
M(k ; n-1,1)(\mathbb{C}) \simeq[\Gamma \backslash \mathscr{D}] .
$$

There is an obvious $*$-variant of this uniformization, which gives

$$
M(\boldsymbol{k} ; n-1,1)^{*}(\mathbb{C}) \simeq\left[\Gamma^{*} \backslash \mathscr{D}\right],
$$

where $\Gamma^{*}$ is the automorphism group of the (parahoric) lattice $L^{*}$ corresponding to the $*$-moduli problem. The lattice $L^{*}$ is uniquely determined up to isomorphism by the condition that there is a chain of inclusions of $O_{k}$-lattices $L^{*} \subset\left(L^{*}\right)^{\vee} \subset$ $(\sqrt{d})^{-1} L^{*}$, with quotient $\left(L^{*}\right)^{\vee} / L^{*}$ of dimension $n-1$ if $n$ is odd and $n-2$ if $n$ is even, when localized at any prime ideal $\mathfrak{p}$ dividing $d$. Here, for an $O_{k}$-lattice $M$ in $V$, we write

$$
M^{\vee}=\left\{x \in V \mid h(x, L) \subset O_{k}\right\}
$$

for the dual lattice.

\section{Special cycles (KM-cycles)}

We continue to assume that the class number of $\boldsymbol{k}$ is one, and recall from [Kudla and Rapoport 2009] the definition of special cycles over $\mathbb{C}$. Let $\left(E, \iota_{0}\right)$ be an elliptic curve with $\mathrm{CM}$ by $O_{k}$ over $\mathbb{C}$, which we fix in what follows. Note that, due to our class number hypothesis, $\left(E, \iota_{0}\right)$ is unique up to isomorphism. We denote its canonical principal polarization by $\lambda_{0}$. For any connected $\mathbb{C}$-scheme $S$ and any $(A, \iota, \lambda) \in \mathcal{M}(\boldsymbol{k} ; n-1,1)(S)$, let

$$
V^{\prime}(A, E)=\operatorname{Hom}_{O_{k}}\left(E_{S}, A\right),
$$

where $E_{S}=E \times_{\mathbb{C}} S$ is the constant elliptic scheme over $S$ defined by $E$. Then $V^{\prime}(A, E)$ is a projective $O_{\boldsymbol{k}}$-module of finite rank with a positive definite $O_{\boldsymbol{k}}$-valued hermitian form given by

$$
h^{\prime}(x, y)=\lambda_{0}^{-1} \circ y^{\vee} \circ \lambda \circ x \in \operatorname{End}_{O_{k}}\left(E_{S}\right)=O_{k} .
$$


For a positive integer $t$, we define the DM- $\operatorname{stack}^{1} \mathscr{L}(t)$ by $\mathscr{L}(t)(S)=\left\{(A, \iota, \lambda ; x) \mid(A, \iota, \lambda) \in \mathcal{M}(\boldsymbol{k} ; n-1,1)(S), x \in V^{\prime}(A, E), h^{\prime}(x, x)=t\right\}$.

Then $\mathscr{L}(t)$ maps by a finite unramified morphism to $\mathcal{M}(\boldsymbol{k} ; n-1,1) \mathbb{C}$, and its image is a divisor in the sense that, locally for the étale topology, it is defined by a nonzero equation.

The cycles $\mathscr{L}(t)$ also admit a complex uniformization. More precisely, under the assumption of the triviality of the class group of $\boldsymbol{k}$, we have

$$
\mathscr{L}(t)(\mathbb{C}) \simeq\left[\Gamma \backslash\left(\coprod_{\substack{x \in L \\ h(x, x)=t}} \mathscr{D}_{x}\right)\right],
$$

where $\mathscr{D}_{x}$ is the set of lines in $\mathscr{D}$ which are perpendicular to $x$.

Again, there is a $*$-variant of these definitions and a corresponding DM-stack $\mathscr{L}(t)^{*}$ above $\mathcal{M}(\boldsymbol{k} ; n-1,1)^{*}$.

\section{Cubic surfaces}

In this paper we consider four occult period mappings. We start with the case of cubic surfaces, following [Allcock et al. 2002]; compare [Beauville 2009]. As explained in the introduction, in these sources, the results are formulated in terms of arithmetic ball quotients; here we use the complex uniformization of the previous two sections to express these results in terms of moduli spaces of Picard type.

Let $S \subset \mathbb{P}^{3}$ be a smooth cubic surface. Let $V$ be a cyclic covering of degree 3 of $\mathbb{P}^{3}$, ramified along $S$. Explicitly, if $S$ is defined by the homogeneous equation of degree 3 in 4 variables

$$
F\left(X_{0}, \ldots, X_{3}\right)=0
$$

then $V$ is defined by the homogeneous equation of degree 3 in 5 variables,

$$
X_{4}^{3}-F\left(X_{0}, \ldots, X_{3}\right)=0 .
$$

Let $\boldsymbol{k}=\mathbb{Q}(\omega), \omega=e^{2 \pi i / 3}$. Then the obvious $\mu_{3}$-action on $V$ determines an action of $O_{k}=\mathbb{Z}[\omega]$ on $H^{3}(V, \mathbb{Z})$. For the (alternating) cup product pairing $\langle$,$\rangle ,$

$$
\langle\omega x, \omega y\rangle=\langle x, y\rangle,
$$

which implies that

$$
\langle a x, y\rangle=\left\langle x, a^{\sigma} y\right\rangle, \quad \text { for all } a \in O_{k} .
$$

${ }^{1}$ This notation differs from that in [Kudla and Rapoport 2009], in that here the special cycles are defined over $\mathbb{C}$, and are considered as lying over $\mathcal{M}(\boldsymbol{k} ; n-1,1)_{\mathbb{C}}$. 
Hence there is a unique $O_{k}$-valued hermitian form $h$ on $H^{3}(V, \mathbb{Z})$ such that

$$
\langle x, y\rangle=\operatorname{tr}\left(\frac{1}{\sqrt{\Delta}} h(x, y)\right),
$$

where the discriminant $\Delta$ of $\boldsymbol{k}$ is equal to -3 in the case at hand. Explicitly,

$$
h(x, y)=\frac{1}{2}(\langle\sqrt{\Delta} x, y\rangle+\langle x, y\rangle \sqrt{\Delta}) .
$$

Furthermore, an $O_{\boldsymbol{k}}$-lattice is self-dual with respect to $\langle$,$\rangle if and only if it is$ self-dual with respect to $h($,$) .$

Fact. $H^{3}(V, \mathbb{Z})$ is a self-dual hermitian $O_{k}$-module of signature $(4,1)$.

As noted above, such a lattice is unique up to isomorphism.

Let

$$
A=A(V)=H^{3}(V, \mathbb{Z}) \backslash H^{3}(V, \mathbb{C}) / H^{2,1}(V)
$$

be the intermediate Jacobian of $V$. Then $A$ is an abelian variety of dimension 5 which is principally polarized by the intersection form. Since the association $V \mapsto(A(V), \lambda)$ is functorial, we obtain an action $\iota$ of $O_{k}$ on $A(V)$.

Theorem 5.1. (i) The object $(A, \iota, \lambda)$ lies in $M(k ; 4,1)(\mathbb{C})$.

(ii) This construction is functorial and compatible with families, and defines a morphism of DM-stacks,

$$
\varphi: \operatorname{Cubics}_{2, \mathbb{C}}^{\circ} \rightarrow M(\boldsymbol{k} ; 4,1)_{\mathbb{C}} .
$$

Here Cubics $_{2, \mathbb{C}}^{\circ}$ denotes the stack parametrizing smooth cubic surfaces up to projective equivalence,

$$
\operatorname{Cubics}_{2, \mathbb{C}}^{\circ}=\left[\mathrm{PGL}_{4} \backslash \mathbb{P} \operatorname{Sym}^{3}\left(\mathbb{C}^{4}\right)^{\circ}\right]
$$

(stack quotient in the orbifold sense).

(iii) The induced morphism on coarse moduli spaces

$$
|\varphi|:\left|\operatorname{Cubics}_{2, \mathbb{C}}^{\circ}\right| \rightarrow|M(\boldsymbol{k} ; 4,1) \mathbb{C}|
$$

is an open embedding. Its image is the complement of the image of the KMcycle $\mathscr{L}(1)$ in $|M(k ; 4,1) \mathbb{C}|$.

Proof. We only comment on the assertions in (ii) and (iii). In (ii), the compatibility with families is always true of Griffiths' intermediate jacobians (which however are abelian varieties only when the Hodge structure is of type $(m+1, m)+(m, m+1))$. This constructs $\varphi$ as a complex-analytic morphism. The algebraicity of $\varphi$ then follows from Borel's theorem [1972] that any analytic family of abelian varieties over a $\mathbb{C}$-scheme is automatically algebraic. The fact that the image is contained in the complement of $\mathscr{L}(1)$ is true because, by the Clemens-Griffiths theory, intermediate 
Jacobians of cubic threefolds are simple as polarized abelian varieties, whereas over $\mathscr{L}(1)$, the polarized abelian varieties split off an elliptic curve. However, the fact that $\mathscr{L}(1)$ makes up the whole complement is surprising and results from the fact that the morphism $\varphi$ extends to an isomorphism from a partial compactification $\left|\mathrm{Cubics}_{2, \mathbb{C}}^{\mathrm{s}}\right|$ of $\left|\mathrm{Cubics}_{2, \mathbb{C}}^{\circ}\right|$ (obtained by adding stable cubics) to $|\mathcal{M}(\boldsymbol{k} ; 4,1) \mathbb{C}|$, such that the complement of $\left|\mathrm{Cubics}_{2, \mathbb{C}}^{\circ}\right|$ in $\left|\mathrm{Cubics}_{2, \mathbb{C}}^{\mathrm{s}}\right|$ is an irreducible divisor; see [Beauville 2009, Propositions 6.7 and 8.2].

Remark 5.2. Let us comment on the stacks aspect of Theorem 5.1. Any automorphism of $S$ is induced by an automorphism of $\mathbb{P}^{3}$, which in turn induces an automorphism of $V$. We therefore obtain a homomorphism $\operatorname{Aut}(S) \rightarrow \operatorname{Aut}(A(V), \iota, \lambda)$. The statement of [Allcock et al. 2002, Theorem 2.20] implies that this homomorphism induces an isomorphism

$$
\operatorname{Aut}(S) \stackrel{\sim}{\longrightarrow} \operatorname{Aut}(A(V), \iota, \lambda) / O_{k}^{\times},
$$

where the units $O_{\boldsymbol{k}}^{\times} \simeq \mu_{6}$ act via $\iota$ on $A(V)$. Indeed, in [Allcock et al. 2002] it is asserted that $\varphi$ is an open immersion of orbifolds Cubics ${ }_{2, \mathbb{C}}^{\circ} \rightarrow[P \Gamma \backslash \mathscr{D}]$, where $P \Gamma=\Gamma / O_{k}^{\times}$; however, we were not able to follow the argument. Note that the orbifold $[P \Gamma \backslash \mathscr{D}]$ is different from $[\Gamma \backslash \mathscr{D}]$, which occurs in Section 3.

\section{Cubic threefolds}

Our next example concerns cubic threefolds, following Allcock et al. [2011] and Looijenga and Swierstra [2007].

Let $T \subset \mathbb{P}^{4}$ be a cubic threefold. Let $V$ be the cyclic covering of degree 3 of $\mathbb{P}^{4}$, ramified in $T$. Then $V$ is a cubic hypersurface in $\mathbb{P}^{5}$ and we define the primitive cohomology as

$$
L=H_{0}^{4}(V, \mathbb{Z})=\left\{x \in H^{4}(V, \mathbb{Z}) \mid(x, \rho)=0\right\},
$$

where $\rho$ is the square of the hyperplane section class. Note that $\operatorname{rk}_{\mathbb{Z}} L=22$. Again, let $\boldsymbol{k}=\mathbb{Q}(\omega)$, with $\omega=e^{2 \pi i / 3}$, so that $L$ becomes an $O_{\boldsymbol{k}}$-module. Now the cup product $\left(\right.$, ) on $H^{4}(V, \mathbb{Z})$ is a perfect symmetric pairing satisfying $(a x, y)=\left(x, a^{\sigma} y\right)$ for $a \in O_{\boldsymbol{k}}$. It induces on $L$ a symmetric bilinear form ( , ) of discriminant 3. We wish to define an alternating pairing $\langle$,$\rangle on L$ satisfying $\langle a x, y\rangle=\left\langle x, a^{\sigma} y\right\rangle$ for $a \in O_{k}$. We do this by giving the associated $O_{k}$-valued hermitian pairing $h($,$) , in the sense$ of (5-1), defined by

$$
h(x, y)=\frac{3}{2}\left((x, y)+(x, \sqrt{\Delta} y) \frac{1}{\sqrt{\Delta}}\right) .
$$

Here the factor $\frac{3}{2}$ is used instead of $\frac{1}{2}$ to have better integrality properties. Set $\pi=\sqrt{\Delta}$. 
Fact. For the pairing (6-2), $L^{\vee}$ contains $\pi^{-1} L$ with $L^{\vee} / \pi^{-1} L \simeq \mathbb{Z} / 3 \mathbb{Z}$.

For this result, see [Allcock et al. 2011, Theorem 2.6 and its proof], as well as [Looijenga and Swierstra 2007, the passage below (2.1)].

Now consider the eigenspace decomposition of $H_{0}^{4}(V, \mathbb{C})$ under $\boldsymbol{k} \otimes \mathbb{C}=\mathbb{C} \oplus \mathbb{C}$.

Fact. The Hodge structure of $H_{0}^{4}(V, \mathbb{R})$ is of type

$$
H_{0}^{4}(V, \mathbb{C})=H^{3,1} \oplus H_{0}^{2,2} \oplus H^{1,3},
$$

with $\operatorname{dim} H^{3,1}=\operatorname{dim} H^{1,3}=1$. Furthermore, the only nontrivial eigenspaces of the generator $\omega$ of $\mu_{3}$ are

$$
\begin{array}{ll}
H_{0}^{4}(V, \mathbb{C})_{\omega}=H^{3,1} \oplus\left(H_{0}^{2,2}\right)_{\omega}, & \text { with } \operatorname{dim}\left(H_{0}^{2,2}\right)_{\omega}=10, \\
H_{0}^{4}(V, \mathbb{C})_{\bar{\omega}}=\left(H_{0}^{2,2}\right)_{\bar{\omega}} \oplus H^{1,3}, & \text { with } \operatorname{dim}\left(H_{0}^{2,2}\right)_{\bar{\omega}}=10 .
\end{array}
$$

(See [Allcock et al. 2011, §2] and [Looijenga and Swierstra 2007, §4], respectively.)

Now set $\Lambda=\pi L^{\vee}$. Then we have the chain of inclusions of $O_{k}$-lattices

$$
\Lambda \subset \Lambda^{\vee} \subset \pi^{-1} \Lambda
$$

where the quotient $\Lambda^{\vee} / \Lambda$ is isomorphic to $(\mathbb{Z} / 3 \mathbb{Z})^{10}$, and where $\pi^{-1} \Lambda / \Lambda^{\vee}$ is isomorphic to $\mathbb{Z} / 3 \mathbb{Z}$. Let

$$
A=\Lambda \backslash H_{0}^{4}(V, \mathbb{C}) / H^{-},
$$

where

$$
H^{-}=H^{3,1} \oplus\left(H_{0}^{2,2}\right)_{\bar{\omega}} .
$$

Note that the map $\Lambda \rightarrow H_{0}^{4}(V, \mathbb{C}) / H^{-}$is an $O_{\boldsymbol{k}}$-linear injection; hence $A$ is a complex torus. In fact, the hermitian form $h$ and its associated alternating form $\langle$,$\rangle define a polarization \lambda$ on $A$. Hence $A$ is an abelian variety of dimension 11 , with an action of $O_{k}$ and a polarization of degree $3^{10}$. In fact, we obtain in this way an object $(A, \iota, \lambda)$ of $\mathcal{M}(\boldsymbol{k} ; 10,1)^{*}(\mathbb{C})$ (see Section 2 for the definition of the $*$-variants of our moduli stacks).

Theorem 6.1. (i) The construction which associates to a smooth cubic $T$ in $\mathbb{P}^{4}$ the object $(A, \iota, \lambda)$ of $\mathcal{M}(\boldsymbol{k} ; 10,1)^{*}(\mathbb{C})$ is functorial and compatible with families, and defines a morphism of DM-stacks

$$
\varphi: \operatorname{Cubics}_{3, \mathbb{C}}^{\circ} \rightarrow M(\boldsymbol{k} ; 10,1)_{\mathbb{C}}^{*} .
$$

(ii) The induced morphism on coarse moduli spaces

$$
|\varphi|:\left|\operatorname{Cubics}_{3, \mathbb{C}}^{\circ}\right| \rightarrow\left|\mathcal{M}(\boldsymbol{k} ; 10,1)_{\mathbb{C}}^{*}\right|
$$

is an open embedding. Its image is the complement of the image of the KMcycle $\mathscr{E}(3)^{*}$ in $\left|\mathcal{M}(\boldsymbol{k} ; 10,1)_{\mathbb{C}}^{*}\right|$. 
Proof. The compatibility with families is due to the fact that the eigenspaces for the $\mu_{3}$-action and the Hodge filtration both vary in a holomorphic way. Point (ii) follows from [Allcock et al. 2011, Theorem 1.1] or [Looijenga and Swierstra 2007, Theorem 3.1].

Remark 6.2. The stack aspect is not treated in these sources. However, it seems reasonable to conjecture that the analogue of (5-3) is also true in this case, that is, that there is an isomorphism

$$
\operatorname{Aut}(T) \stackrel{\sim}{\longrightarrow} \operatorname{Aut}(A, \iota, \lambda) / O_{k}^{\times},
$$

where $(A, \iota, \lambda)$ is the object of $M(\boldsymbol{k} ; 10,1)_{\mathbb{C}}^{*}$ attached to $T$.

Remark 6.3. The construction of the rational Hodge structure $H^{1}(A, \mathbb{Q})$ from $H_{0}^{4}(V, \mathbb{Q})$ is a very special case of a general construction due to van Geemen [2001]. More precisely, it arises (up to Tate twist) as the inverse half-twist in the sense of [van Geemen 2001] of the Hodge structure $H_{0}^{4}(V, \mathbb{Q})$ with complex multiplication by $\boldsymbol{k}$. The half-twist construction attaches to a rational Hodge structure $V$ of weight $w$ with complex multiplication by a CM-field $\boldsymbol{k}$ a rational Hodge structure of weight $w+1$. More precisely, if $\Sigma$ is a fixed half-system of complex embeddings of $\boldsymbol{k}$, then van Geemen defines a new Hodge structure on $V$ by setting

$$
V_{\text {new }}^{r, s}=V_{\Sigma}^{r-1, s} \oplus V_{\bar{\Sigma}}^{r, s-1},
$$

where $V_{\Sigma}$ (resp. $V_{\bar{\Sigma}}$ ) denotes the sum of the eigenspaces for the $\boldsymbol{k}$-action corresponding to the complex embeddings in $\Sigma$ (resp. in $\bar{\Sigma}$ ).

\section{Curves of genus 3}

Our third example concerns the moduli space of curves of genus 3 following Kondō [2000].

Let $C$ be a non-hyperelliptic smooth projective curve of genus 3. The canonical system embeds $C$ as a quartic curve in $\mathbb{P}^{2}$. Let $X(C)$ be the $\mu_{4}$-covering of $\mathbb{P}^{2}$ ramified in $C$. Then the quartic $X(C) \subset \mathbb{P}^{3}$ is a K3-surface with an automorphism $\tau$ of order 4 and hence an action of $\mu_{4}$. Let

$$
L=\left\{x \in H^{2}(X(C), \mathbb{Z}) \mid \tau^{2}(x)=-x\right\} .
$$

Let $\boldsymbol{k}=\mathbb{Q}(i)$ be the Gaussian field.

Fact. $L$ is a free $\mathbb{Z}$-module of rank 14 . The restriction (, ) of the symmetric cup product pairing to $L$ has discriminant $2^{8}$; more precisely, for the dual lattice $L^{*}$ for the symmetric pairing,

$$
L^{*} / L \cong(\mathbb{Z} / 2)^{8} \text {. }
$$

(See [Kondō 2000, top of p. 222].) 
Now consider the eigenspace decomposition of $L_{\mathbb{C}}=L \otimes \mathbb{C}$ under $\boldsymbol{k} \otimes \mathbb{C}=\mathbb{C} \oplus \mathbb{C}$, where $i \otimes 1$ acts via $\tau$.

Fact. The induced Hodge structure on $L_{\mathbb{C}}$ is of type

$$
L_{\mathbb{C}}=L^{2,0} \oplus L^{1,1} \oplus L^{0,2},
$$

with $\operatorname{dim} L^{2,0}=\operatorname{dim} L^{0,2}=1$. Furthermore, the only nontrivial eigenspaces of $\tau$ are

$$
\begin{array}{rlrl}
\left(L_{\mathbb{C}}\right)_{i} & =L^{2,0} \oplus\left(L^{1,1}\right)_{i}, & & \text { with } \operatorname{dim}\left(L^{1,1}\right)_{i}=6, \\
\left(L_{\mathbb{C}}\right)_{-i} & =\left(L^{1,1}\right)_{-i} \oplus L^{0,2}, & \text { with } \operatorname{dim}\left(L^{1,1}\right)_{-i}=6 .
\end{array}
$$

We define an $O_{\boldsymbol{k}}$-valued hermitian pairing $h$ on $L_{\mathbb{Q}}$ by setting

$$
h(x, y)=(x, y)+(x, \tau y) i .
$$

Then it is easy to see that the dual lattice $L^{\vee}$ of $L$ for the hermitian form $h$ is the same as the dual lattice $L^{*}$ for the symmetric form.

Now set $\Lambda=\pi L^{\vee}$, where $\pi=1+i$. Then we obtain a chain of inclusions of $O_{k}$-lattices

$$
\Lambda \subset \Lambda^{\vee} \subset \pi^{-1} \Lambda
$$

where the quotient $\Lambda^{\vee} / \Lambda$ is isomorphic to $(\mathbb{Z} / 2 \mathbb{Z})^{6}$, and where $\pi^{-1} \Lambda / \Lambda^{\vee}$ is isomorphic to $\mathbb{Z} / 2 \mathbb{Z}$.

Let

$$
A=\Lambda \backslash L_{\mathbb{C}} / L^{-},
$$

where

$$
L^{-}=L^{2,0} \oplus\left(L^{1,1}\right)_{-i}
$$

Note that the map $\Lambda \rightarrow L_{\mathbb{C}} / L^{-}$is an $O_{\boldsymbol{k}}$-linear injection; hence $A$ is a complex torus. In fact, the hermitian form $h$ and its associated alternating form $\langle$,$\rangle define a$ polarization $\lambda$ on $A$. Hence $A$ is an abelian variety of dimension 7 , with an action of $O_{k}$ and a polarization of degree $2^{6}$. In fact, we obtain in this way an object $(A, \iota, \lambda)$ of $\mathcal{M}(\boldsymbol{k} ; 6,1)^{*}(\mathbb{C})$. Now [Kondō 2000, Theorem 2.5] implies the following theorem.

Theorem 7.1. (i) The construction which associates to a non-hyperelliptic curve of genus 3 the object $(A, \iota, \lambda)$ of $\mathcal{M}(\boldsymbol{k} ; 6,1)^{*}(\mathbb{C})$ is functorial and compatible with families, and defines a morphism of DM-stacks

$$
\varphi: \mathcal{N}_{3, \mathbb{C}}^{\circ} \rightarrow M(\boldsymbol{k} ; 6,1)_{\mathbb{C}}^{*}
$$

Here $\mathcal{N}_{3, \mathbb{C}}^{\circ}$ denotes the stack of smooth non-hyperelliptic curves of genus 3 , that is, of smooth non-hyperelliptic quartics in $\mathbb{P}^{2}$ up to projective equivalence. 
(ii) The induced morphism on coarse moduli schemes $|\varphi|:\left|\mathcal{N}_{3, \mathbb{C}}^{\circ}\right| \rightarrow\left|\mathcal{M}(\boldsymbol{k} ; 6,1)_{\mathbb{C}}^{*}\right|$ is an open embedding. Its image is the complement of the image of the KM-cycle $\mathscr{L}(2)^{*}$ in $\left|M(k ; 6,1)_{\mathbb{C}}^{*}\right|$.

Remark 7.2. Again, the stack aspect is not treated in [Kondō 2000]. It seems reasonable to conjecture that the analogue of (5-3) is also true in this case, that is, that there is an isomorphism

$$
\operatorname{Aut}(C) \stackrel{\sim}{\longrightarrow} \operatorname{Aut}(A, \iota, \lambda) / O_{k}^{\times},
$$

where $(A, \iota, \lambda)$ is the object of $M(\boldsymbol{k} ; 6,1)_{\mathbb{C}}^{*}$ attached to $C$, and where $O_{\boldsymbol{k}}^{\times}=\mu_{4}$.

\section{Curves of genus 4}

Our final example concerns the moduli space of curves of genus 4 and is also due to Kondō [2002].

Let $C$ be a non-hyperelliptic curve of genus 4 . The canonical system embeds $C$ into $\mathbb{P}^{3}$. More precisely, $C$ is the intersection of a smooth cubic surface $S$ and a quartic $Q$ which is either smooth or a quadratic cone. Furthermore, $Q$ is uniquely determined by $C$. Let $X$ be a cyclic cover of degree 3 over $Q$ branched along $C$ (if $Q$ is singular, we take the minimal resolution of the singularities; see [Kondō 2002]). Then $X$ is a K3-surface with an action of $\mu_{3}$. Let

$$
L=\left(H^{2}(X, \mathbb{Z})^{\mu_{3}}\right)^{\perp}
$$

be the orthogonal complement of the invariants of this action in $H^{2}(X, \mathbb{Z})$, equipped with the symmetric form ( , ) obtained by restriction.

Fact. $L$ is a free $\mathbb{Z}$-module of rank 20 , with dual $L^{*}$ for the symmetric form satisfying

$$
L^{*} / L \simeq(\mathbb{Z} / 3 \mathbb{Z})^{2} .
$$

(See [Kondō 2002, top of p. 386].)

For $\boldsymbol{k}=\mathbb{Q}(\omega), \omega=e^{2 \pi i / 3}$, we again define an alternating form $\langle$,$\rangle through its$ associated $O_{k}$-valued hermitian form $h$. Using the action of $O_{k}$ on $L$, we set

$$
h(x, y)=\frac{3}{2}\left((x, y)+(x, \sqrt{\Delta} y) \frac{1}{\sqrt{\Delta}}\right) .
$$

Set $\pi=\sqrt{\Delta}$.

Fact. For the hermitian pairing (8-1), $L^{\vee}$ is an over-lattice of $\pi^{-1} L$ with

$$
L^{\vee} / \pi^{-1} L \simeq(\mathbb{Z} / 3 \mathbb{Z})^{2} .
$$

Now consider the eigenspace decomposition of $L \otimes \mathbb{C}$ under $\boldsymbol{k} \otimes \mathbb{C}=\mathbb{C} \oplus \mathbb{C}$. 
Fact. The induced Hodge structure on $L_{\mathbb{C}}$ is of type

$$
L_{\mathbb{C}}=L^{2,0} \oplus L^{1,1} \oplus L^{0,2},
$$

with $\operatorname{dim} L^{2,0}=\operatorname{dim} L^{0,2}=1$. Furthermore, the only nontrivial eigenspaces of $\mu_{3}$ are

$$
\begin{array}{ll}
\left(L_{\mathbb{C}}\right)_{\omega}=L^{2,0} \oplus\left(L^{1,1}\right)_{\omega}, & \text { with } \operatorname{dim}\left(L^{1,1}\right)_{\omega}=9, \\
\left(L_{\mathbb{C}}\right)_{\bar{\omega}}=\left(L^{1,1}\right)_{\bar{\omega}} \oplus L^{0,2}, & \text { with } \operatorname{dim}\left(L^{1,1}\right)_{\bar{\omega}}=9 .
\end{array}
$$

Now set $\Lambda=\pi L^{\vee}$. Then we have the chain of inclusions of $O_{k}$-lattices

$$
\Lambda \subset \Lambda^{\vee} \subset \pi^{-1} \Lambda
$$

where the quotient $\Lambda^{\vee} / \Lambda$ is isomorphic to $(\mathbb{Z} / 3 \mathbb{Z})^{8}$, and where $\pi^{-1} \Lambda / \Lambda^{\vee}$ is isomorphic to $(\mathbb{Z} / 3 \mathbb{Z})^{2}$.

Let

$$
A=\Lambda \backslash L_{\mathbb{C}} / L^{-},
$$

where

$$
L^{-}=L^{2,0} \oplus\left(L^{1,1}\right) \bar{\omega} .
$$

Then the map $\Lambda \rightarrow L_{\mathbb{C}} / L^{-}$is an $O_{\boldsymbol{k}}$-linear injection; hence $A$ is a complex torus. In fact, the hermitian form $h$ and its associated alternating form $\langle$,$\rangle define a$ polarization $\lambda$ on $A$. Hence $A$ is an abelian variety of dimension 10, with an action of $O_{k}$ and a polarization of degree $3^{8}$. In fact, we obtain in this way an object $(A, \iota, \lambda)$ of $\mathcal{M}(\boldsymbol{k} ; 9,1)^{*}(\mathbb{C})$.

Theorem 8.1. (i) The construction which associates to a non-hyperelliptic curve of genus 4 the object $(A, \iota, \lambda)$ of $\mathcal{M}(\boldsymbol{k} ; 9,1)^{*}(\mathbb{C})$ is functorial and compatible with families, and defines a morphism of DM-stacks

$$
\varphi: \mathcal{N}_{4, \mathbb{C}}^{\circ} \rightarrow M(\boldsymbol{k} ; 9,1)_{\mathbb{C}}^{*}
$$

Here $\mathcal{N}_{4, \mathbb{C}}^{\circ}$ denotes the stack of smooth non-hyperelliptic curves of genus 4.

(ii) The induced morphism on coarse moduli schemes $|\varphi|:\left|\mathcal{N}_{4, \mathbb{C}}^{\circ}\right| \rightarrow\left|\mathcal{M}(\boldsymbol{k} ; 9,1)_{\mathbb{C}}^{*}\right|$ is an open embedding. Its image is the complement of the image of the KM-cycle $\mathscr{L}(2)^{*}$ in $\left|\mathcal{M}(\boldsymbol{k} ; 9,1)_{\mathbb{C}}^{*}\right|$.

Remark 8.2. Again, the stack aspect is not treated in [Kondō 2002]. It seems reasonable to conjecture that the analogue of (5-3) is also true in this case, that is, that there is an isomorphism

$$
\operatorname{Aut}(C) \stackrel{\sim}{\longrightarrow} \operatorname{Aut}(A, \iota, \lambda) / O_{k}^{\times},
$$

where $(A, \iota, \lambda)$ is the object of $\mathcal{M}(\boldsymbol{k} ; 9,1)_{\mathbb{C}}^{*}$ attached to $C$, and where $O_{\boldsymbol{k}}^{\times}=\mu_{6}$. 


\section{Descent}

In all four cases discussed above, we obtain morphisms over $\mathbb{C}$ between DM-stacks defined over $\boldsymbol{k}$. These morphisms are constructed using transcendental methods. In this section we will show that these morphisms are in fact defined over $\boldsymbol{k}$. The argument is modeled on Deligne's solution [1972] of the analogous problem for complete intersections of Hodge level one, where he shows that the corresponding family of intermediate jacobians is an abelian scheme over the moduli scheme over $\mathbb{Q}$ of complete intersections of given multidegree.

In our discussion below, to simplify notations, we will deal with the case of cubic threefolds, as explained in Section 6; the other cases are completely analogous. Below we will shorten the notation Cubics $_{3}^{\circ}$ to $\mathscr{C}$, and consider this as a DMstack over Spec $\boldsymbol{k}$. Let $v: V \rightarrow \mathscr{C}$ be the universal family of cubic threefolds, and let $a: A \rightarrow \mathscr{C}_{\mathbb{C}}$ be the polarized family of abelian varieties constructed from $V$ in Section 6. Hence $A$ is the pullback of the universal abelian scheme over $M(\boldsymbol{k} ; 10,1)_{\mathbb{C}}^{*}$ under the morphism $\varphi: \mathscr{C}_{\mathbb{C}} \rightarrow M(\boldsymbol{k} ; 10,1)_{\mathbb{C}}^{*}$.

Lemma 9.1. Let $b: B \rightarrow \mathscr{C}_{\mathbb{C}}$ be a polarized abelian scheme with $O_{\boldsymbol{k}}$-action, which is the pullback under a morphism $\psi: \mathscr{C}_{\mathbb{C}} \rightarrow M(\boldsymbol{k} ; 10,1)_{\mathbb{C}}^{*}$ of the universal abelian scheme, and such that there exists $\ell$ and an $O_{k}$-linear isomorphism of lisse $\ell$-adic sheaves on $\mathfrak{b}_{\mathbb{C}}$,

$$
\alpha_{\ell}: R^{1} a_{*} \mathbb{Z}_{\ell} \simeq R^{1} b_{*} \mathbb{Z}_{\ell},
$$

compatible with the Riemann forms on source and target. Then there exists a unique isomorphism $\alpha: A \rightarrow B$ that induces $\alpha_{\ell}$. This isomorphism is compatible with polarizations.

To prove this, we are going to use the following lemma. In it, we denote by $\Lambda$ the hermitian $O_{\boldsymbol{k}}$-module $H^{1}\left(A_{s}, \mathbb{Z}\right)$, for $s \in \mathscr{C}_{\mathbb{C}}$ a fixed base point. Recall from Section 6 that there is a chain of inclusions $\Lambda \subset \Lambda^{\vee} \subset \pi^{-1} \Lambda$, where $\pi=\sqrt{-3}$ is a generator of the unique prime ideal of $O_{k}$ dividing 3 .

Lemma 9.2. Let $s \in \mathscr{C}_{\mathbb{C}}$ be the chosen base point.

(i) The monodromy representation $\rho_{A}: \pi_{1}\left(\mathscr{C}_{\mathbb{C}}, s\right) \rightarrow \mathrm{GL}_{k}\left(\Lambda \otimes_{O_{k}} k\right)$ is absolutely irreducible.

(ii) For every prime ideal $\mathfrak{p}$ prime to 3 , the monodromy representation

$$
\pi_{1}\left(\mathscr{C}_{\mathbb{C}}, s\right) \rightarrow \mathrm{GL}_{\kappa(\mathfrak{p})}(\Lambda / \mathfrak{p} \Lambda)
$$

is absolutely irreducible.

(iii) For the unique prime ideal $\mathfrak{p}=(\pi)$ lying over 3 , the monodromy representation $\pi_{1}\left(\mathscr{C}_{\mathbb{C}}, s\right) \rightarrow \mathrm{GL}_{\kappa(\mathfrak{p})}(\Lambda / \mathfrak{p} \Lambda)$ is not absolutely irreducible, but there is a unique 
nontrivial stable subspace, namely, the 10-dimensional image of $\pi \Lambda^{\vee}$ in $\Lambda / \pi \Lambda$.

Proof. The monodromy representations in question are induced by the composition of homomorphisms

$$
\pi_{1}\left(\mathscr{C}_{\mathbb{C}}, s\right) \longrightarrow \pi_{1}\left(M(\boldsymbol{k} ; 10,1)_{\mathbb{C}}^{*}, \varphi(s)\right) \longrightarrow \mathrm{GL}_{O_{k}}\left(H^{1}\left(A_{s}, \mathbb{Z}\right)\right) .
$$

Here by Theorem 6.1, and using complex uniformization (see Section 3), the first homomorphism is induced by the inclusion of connected spaces

$$
\iota: \mathscr{D} \backslash\left(\bigcup_{\substack{x \in L \\ h(x, x)=3}} \mathscr{D}_{x}\right) \hookrightarrow \mathscr{D},
$$

followed by quotienting out by the free action of $\Gamma^{*}$. Since $\mathscr{D}$ is simply connected, it follows that $\pi_{1}\left(M(\boldsymbol{k} ; 10,1)_{\mathbb{C}}^{*}, \varphi(s)\right)=\Gamma^{*}$ and that the first homomorphism in (9-1) is surjective. Now, $\Gamma^{*}$ can be identified with the group of unitary automorphisms of the parahoric lattice $\Lambda$, and it is elementary that the representations of $\Gamma^{*}$ on $\Lambda \otimes_{O_{k}} \boldsymbol{k}$ and on $\Lambda / \mathfrak{p} \Lambda$ for $\mathfrak{p}$ prime to 3 are absolutely irreducible (the latter since $\Lambda^{\vee} \otimes \mathbb{Z}_{\ell}=\Lambda \otimes \mathbb{Z}_{\ell}$ for $\ell \neq 3$ ). The statement (iii) is proved in the same way.

Proof of Lemma 9.1. Let us compare the monodromy representations

$$
\begin{aligned}
& \rho_{A}: \pi_{1}\left(\mathscr{C}_{\mathbb{C}}, s\right) \rightarrow \operatorname{GL}_{O_{k}}\left(H^{1}\left(A_{s}, \mathbb{Z}\right)\right), \\
& \rho_{B}: \pi_{1}\left(\mathscr{C}_{\mathbb{C}}, s\right) \rightarrow \operatorname{GL}_{O_{k}}\left(H^{1}\left(B_{s}, \mathbb{Z}\right)\right) .
\end{aligned}
$$

By hypothesis, these representations are isomorphic after tensoring with $\mathbb{Z}_{\ell}$. Hence, they are also isomorphic after tensoring with $\boldsymbol{k}$. Hence there exists a $\pi_{1}\left(\mathscr{C}_{\mathbb{C}}, s\right)$ equivariant $\boldsymbol{k}$-linear isomorphism

$$
\beta: H^{1}\left(A_{s}, \mathbb{Q}\right) \simeq H^{1}\left(B_{s}, \mathbb{Q}\right) .
$$

By the irreducibility of the representation of $\pi_{1}\left(\mathscr{C}_{\mathbb{C}}, s\right)$ in $H^{1}\left(A_{s}, \mathbb{Q}\right), \beta$ is unique up to a scalar in $\boldsymbol{k}^{\times}$. Let us compare the $O_{\boldsymbol{k}}$-lattices $\beta^{-1}\left(H^{1}\left(B_{s}, \mathbb{Z}\right)\right)$ and $H^{1}\left(A_{s}, \mathbb{Z}\right)$. Since we are assuming that $O_{k}$ is a PID, after replacing $\beta$ by a multiple $\beta_{\odot}=c \beta$, we may assume that $L_{B}=\beta_{\odot}^{-1}\left(H^{1}\left(B_{s}, \mathbb{Z}\right)\right)$ is a primitive $O_{k}$-sublattice in $\Lambda=$ $H^{1}\left(A_{s}, \mathbb{Z}\right)$. Let $\mathfrak{p}$ be a prime ideal in $O_{k}$, and let us consider the image of $L_{B}$ in $\Lambda / \mathfrak{p} \Lambda$. Since $L_{B}$ is primitive in $\Lambda$, this image is nonzero. If $\mathfrak{p}$ is prime to 3 , the irreducibility statement in (ii) of Lemma 9.2 implies that this image is everything, and hence $L_{B} \otimes O_{k, \mathfrak{p}}=\Lambda \otimes O_{k, \mathfrak{p}}$ in this case.

To handle the prime ideal $\mathfrak{p}=(\pi)$ over 3 , we use the polarizations. By the irreducibility statement in (i) of Lemma 9.2, the polarization forms on $H^{1}\left(A_{s}, \mathbb{Q}\right)$ and on $H^{1}\left(B_{s}, \mathbb{Q}\right)$ differ by a scalar in $\mathbb{Q}^{\times}$under the isomorphism $\beta_{\mathbb{O}}$. Now, by hypothesis on $B$, with respect to the polarization form on $H^{1}\left(B_{s}, \mathbb{Q}\right)$, we have a chain of inclusions $L_{B} \subset L_{B}^{\vee} \subset \pi^{-1} L_{B}$ with respective quotients of dimension 10 
and 1 over $\mathbb{F}_{p}$, just as for $\Lambda$. Since the two polarization forms differ by a scalar, this excludes the possibility that the image of $L_{B}$ in $\Lambda / \pi \Lambda$ be nontrivial. It follows that $L_{B}=\Lambda$.

Furthermore, the isomorphism $\beta_{\odot}$ is unique up to a unit in $O_{k}^{\times}$, and it is an isometry with respect to both polarization forms. Now, by [Deligne 1971, 4.4.11 and 4.4.12], $\beta_{\odot}$ is induced by an isomorphism of polarized abelian schemes. Finally, $\beta_{\odot} \otimes_{\mathbb{Z}} \mathbb{Z}_{\ell}=\alpha_{\ell}$ up to a unit, since these homomorphisms differ by a scalar and both preserve the Riemann forms.

The uniqueness of $\alpha$ follows from Serre's Lemma.

Now Lemma 9.1 implies that over any field extension $k^{\prime}$ of $\boldsymbol{k}$ inside $\mathbb{C}$, there exists at most one polarized abelian variety $b: B \rightarrow \mathscr{C}_{k^{\prime}}$ obtained by pull-back from the universal abelian variety over $\mathcal{M}(\boldsymbol{k} ; 10,1)^{*}$, equipped with an $O_{\boldsymbol{k}}$-linear isomorphism of lisse $\ell$-adic sheaves over $\mathscr{C}_{\mathbb{C}}$

$$
R^{1} a_{*} \mathbb{Z}_{\ell} \simeq R^{1} b_{\mathbb{C} *} \mathbb{Z}_{\ell}
$$

preserving the Riemann forms. By the argument in [Deligne 1972, 2.2], this implies that, in fact, $B$ exists (since it does for $k^{\prime}=\mathbb{C}$ ). Hence the morphism $\varphi$ is defined over $\boldsymbol{k}$. Put otherwise, for any $\boldsymbol{k}$-automorphism $\tau$ of $\mathbb{C}$, the conjugate embedding $\varphi^{\tau}$, which corresponds to the conjugate $(A, \iota, \lambda)^{\tau}$, is equal to $\varphi$; hence $\varphi$ is defined over $\boldsymbol{k}$.

Conjecture 9.3. In all four cases above, the morphisms $\varphi$ can be extended over $O_{k}\left[\Delta^{-1}\right]$.

Since we circulated a first version of our paper, this has been proved by J. Achter [2012] in the case of cubic surfaces.

\section{Concluding remarks}

Remark 10.1. In all four cases, the complement of $\operatorname{Im}(|\varphi|)$ is identified with a certain KM-divisor. In fact, for other KM-divisors, the intersection with $\operatorname{Im}(|\varphi|)$ sometimes has a geometric interpretation. For example, in the case of cubic surfaces, the intersection of $\operatorname{Im}(|\varphi|)$ with the image of the KM-divisor $\mathscr{L}(2)$ in $\left|\mathcal{M}(\boldsymbol{k} ; 4,1)_{\mathbb{C}}\right|$ can be identified with the locus of cubic surfaces admitting Eckardt points; see [Dolgachev et al. 2005, Theorem 8.10]. Similarly, in the case of curves of genus 3, the intersection of $\operatorname{Im}(|\varphi|)$ with the image of $\mathscr{L}(t)^{*}$ in $\left|\mathcal{M}(\boldsymbol{k} ; 6,1)_{\mathbb{C}}^{*}\right|$ can be identified with the locus of curves $C$ where the K3-surface $X(C)$ admits a "splitting curve" of a certain degree depending on $t$; see [Artebani 2008, Theorem 4.6].

Remark 10.2. In [Dolgachev and Kondō 2007; Dolgachev et al. 2005; Matsumoto et al. 1992], occult period morphisms are often set in comparison with the DeligneMostow theory, which establishes a relation between configuration spaces (for 
example, of points in the projective line) and quotients of the complex unit ball by complex reflection groups, via monodromy groups of hypergeometric equations. This aspect of these examples has been suppressed entirely here. Also, it should be mentioned that there are other ways of constructing the period map for cubic surfaces; see, e.g., [Dolgachev and Kondō 2007; Dolgachev et al. 2005].

Remark 10.3. Let us return to Section 3. There we had fixed a hermitian vector space $(V,()$,$) over \boldsymbol{k}$ of signature $(n-1,1)$. Let $V_{0}$ be the underlying $\mathbb{Q}$-vector space, with the symmetric pairing defined by

$$
s(x, y)=\operatorname{tr}(h(x, y)) .
$$

Then $s$ has signature $(2(n-1), 2)$, and we obtain an embedding of $\mathrm{U}(V)$ into $\mathrm{O}\left(V_{0}\right)$. This also induces an embedding of symmetric spaces,

$$
\mathscr{D} \hookrightarrow \mathscr{D}_{\mathrm{O}},
$$

where, as before, $\mathscr{D}$ is the space of negative (complex) lines in $\left(V_{\mathbb{R}}, \square_{0}\right)$, and where $\mathscr{D}_{\mathrm{O}}$ is the space of oriented negative 2-planes in $V_{\mathbb{R}}$. The image of (10-1) is precisely the set of negative 2-planes that are stable by $\rrbracket_{0}$. In the cases of the Gauss field and the Eisenstein field, this invariance is equivalent to being stable under the action of $\mu_{4}$ or $\mu_{6}$, respectively. Hence in these two cases, the image of (10-1) can also be identified with the fixed point locus of $\mu_{4}$ or $\mu_{6}$, respectively, in $\mathscr{D}_{\mathrm{O}}$.

Remark 10.4. By going through the tables in [Rapoport 1972, §2], one sees that there is no further example of an occult period map of the type above which embeds the moduli stack of hypersurfaces of suitable degree and dimension into a Picard type moduli stack of abelian varieties. Note, however, that, in the case of curves of genus 4 , the source of the hidden period morphism is a moduli stack of complete intersections of a certain multidegree of dimension one, and there may be more examples of this type.

\section{Acknowledgements}

We thank B. van Geemen, D. Huybrechts and E. Looijenga for very helpful discussions. We also thank J. Achter for keeping us informed about his progress in proving our conjecture in Section 9 in some cases. Finally, we thank the referee who alerted us to a mistake concerning the stacks aspect of period maps.

\section{References}

[Achter 2012] J. Achter, "Arithmetic Torelli maps for cubic surfaces and threefolds", preprint, 2012. arXiv 1005.2131 
[Allcock et al. 2002] D. Allcock, J. A. Carlson, and D. Toledo, "The complex hyperbolic geometry of the moduli space of cubic surfaces", J. Algebraic Geom. 11:4 (2002), 659-724. MR 2003m:32011 Zbl 1080.14532

[Allcock et al. 2011] D. Allcock, J. A. Carlson, and D. Toledo, The moduli space of cubic threefolds as a ball quotient, Mem. Amer. Math. Soc. 985, Amer. Math. Soc., Providence, 2011. MR 2012b:32021

[Artebani 2008] M. Artebani, "Heegner divisors in the moduli space of genus three curves", Trans. Amer. Math. Soc. 360:3 (2008), 1581-1599. MR 2008j:14068 Zbl 1149.14028

[Beauville 2009] A. Beauville, "Moduli of cubic surfaces and Hodge theory (after Allcock, Carlson, Toledo)", pp. 445-466 in Géométries à courbure négative ou nulle, groupes discrets et rigidités (Grenoble, 2004), edited by L. Bessières et al., Sémin. Congr. 18, Soc. Math. France, Paris, 2009. MR 2011g:32022 Zbl 1195.14050

[Borel 1972] A. Borel, "Some metric properties of arithmetic quotients of symmetric spaces and an extension theorem", J. Differential Geometry 6 (1972), 543-560. MR 49 \#3220 Zbl 0249.32018

[Deligne 1971] P. Deligne, “Théorie de Hodge, II”, Inst. Hautes Études Sci. Publ. Math. 40 (1971), 5-57. MR 58 \#16653a Zbl 0219.14007

[Deligne 1972] P. Deligne, "Les intersections complètes de niveau de Hodge un", Invent. Math. 15 (1972), 237-250. MR 46 \#189 Zbl 0219.14008

[Dolgachev and Kondō 2007] I. V. Dolgachev and S. Kondō, "Moduli of K3 surfaces and complex ball quotients", pp. 43-100 in Arithmetic and geometry around hypergeometric functions (Istanbul, 2005), edited by R.-P. Holzapfel et al., Progr. Math. 260, Birkhäuser, Basel, 2007. MR 2008e:14053 Zbl 1124.14032

[Dolgachev et al. 2005] I. V. Dolgachev, B. van Geemen, and S. Kondō, "A complex ball uniformization of the moduli space of cubic surfaces via periods of K3 surfaces", J. Reine Angew. Math. 588 (2005), 99-148. MR 2006h:14051 Zbl 1090.14010

[van Geemen 2001] B. van Geemen, "Half twists of Hodge structures of CM-type", J. Math. Soc. Japan 53:4 (2001), 813-833. MR 2002i:14012 Zbl 1074.14509

[Kondō 2000] S. Kondō, "A complex hyperbolic structure for the moduli space of curves of genus three”, J. Reine Angew. Math. 525 (2000), 219-232. MR 2001j:14039 Zbl 0990.14007

[Kondō 2002] S. Kondō, “The moduli space of curves of genus 4 and Deligne-Mostow's complex reflection groups", pp. 383-400 in Algebraic geometry 2000, Azumino (Nagano, 2000), edited by S. Usui et al., Adv. Stud. Pure Math. 36, Math. Soc. Japan, Tokyo, 2002. MR 2004h:14033 Zbl 1043.14005

[Kudla and Rapoport 2009] S. S. Kudla and M. Rapoport, "Special cycles on unitary Shimura varieties, II: Global theory”, preprint, 2009. arXiv 0912.3758

[Looijenga and Swierstra 2007] E. Looijenga and R. Swierstra, "The period map for cubic threefolds", Compos. Math. 143:4 (2007), 1037-1049. MR 2008f:32015 Zbl 1120.14007

[Looijenga and Swierstra 2008] E. Looijenga and R. Swierstra, "On period maps that are open embeddings", J. Reine Angew. Math. 617 (2008), 169-192. MR 2010a:32030 Zbl 1161.14007

[Matsumoto et al. 1992] K. Matsumoto, T. Sasaki, and M. Yoshida, "The monodromy of the period map of a 4-parameter family of $K 3$ surfaces and the hypergeometric function of type $(3,6)$ ", Internat. J. Math. 3:1 (1992), 164. MR 93a:33029 Zbl 0763.32016

[Rapoport 1972] M. Rapoport, "Complément à l'article de P. Deligne "La conjecture de Weil pour les surfaces K3"”, Invent. Math. 15 (1972), 227-236. MR 46 \#9046 Zbl 0228.14014

Received February 28, 2012. 
STEPHEN KUdLA

DEPARTMENT OF MATHEMATICS

UNIVERSITY OF TORONTO

40 St. George St., BA6290

TORONTO, ON M5S 2E4

CANADA

skudla@math.toronto.edu

MichAEL RAPOPORT

MATHEMATISCHES INSTITUT

UNIVERSITÄT BONN

ENDENICHER ALLEE 60

53115 BONN

GERMANY

rapoport@math.uni-bonn.de 


\title{
PACIFIC JOURNAL OF MATHEMATICS
}

\author{
http://pacificmath.org
}

Founded in 1951 by E. F. Beckenbach (1906-1982) and F. Wolf (1904-1989)

\section{EDITORS}

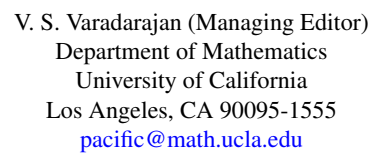

Don Blasius

Department of Mathematics University of California

Los Angeles, CA 90095-1555

blasius@math.ucla.edu

Robert Finn

Department of Mathematics

Stanford University

Stanford, CA 94305-2125

finn@math.stanford.edu

Alexander Merkurjev

Department of Mathematics

University of California

Los Angeles, CA 90095-1555

merkurev@math.ucla.edu

\author{
Vyjayanthi Chari \\ Department of Mathematics \\ University of California \\ Riverside, CA 92521-0135 \\ chari@math.ucr.edu \\ Kefeng Liu \\ Department of Mathematics \\ University of California \\ Los Angeles, CA 90095-1555 \\ liu@math.ucla.edu \\ Sorin Popa \\ Department of Mathematics \\ University of California \\ Los Angeles, CA 90095-1555 \\ popa@math.ucla.edu \\ Paul Yang \\ Department of Mathematics \\ Princeton University \\ Princeton NJ 08544-1000 \\ yang@math.princeton.edu
}

\section{PRODUCTION}

Silvio Levy, Scientific Editor, pacific@math.berkeley.edu

\section{SUPPORTING INSTITUTIONS}

ACADEMIA SINICA, TAIPEI

CALIFORNIA INST. OF TECHNOLOGY

INST. DE MATEMÁTICA PURA E APLICADA

KEIO UNIVERSITY

MATH. SCIENCES RESEARCH INSTITUTE

NEW MEXICO STATE UNIV.

OREGON STATE UNIV.

\author{
STANFORD UNIVERSITY \\ UNIV. OF BRITISH COLUMBIA \\ UNIV. OF CALIFORNIA, BERKELEY \\ UNIV. OF CALIFORNIA, DAVIS \\ UNIV. OF CALIFORNIA, LOS ANGELES \\ UNIV. OF CALIFORNIA, RIVERSIDE \\ UNIV. OF CALIFORNIA, SAN DIEGO \\ UNIV. OF CALIF., SANTA BARBARA
}

\author{
Daryl Cooper \\ Department of Mathematics \\ University of California \\ Santa Barbara, CA 93106-3080 \\ cooper@math.ucsb.edu \\ Jiang-Hua Lu \\ Department of Mathematics \\ The University of Hong Kong \\ Pokfulam Rd., Hong Kong \\ jhlu@maths.hku.hk
}

\section{Jie Qing}

Department of Mathematics

University of California

Santa Cruz, CA 95064

qing@cats.ucsc.edu

These supporting institutions contribute to the cost of publication of this Journal, but they are not owners or publishers and have no responsibility for its contents or policies.

See inside back cover or pacificmath.org for submission instructions.

The subscription price for 2012 is US \$420/year for the electronic version, and \$485/year for print and electronic.

Subscriptions, requests for back issues from the last three years and changes of subscribers address should be sent to Pacific Journal of Mathematics, P.O. Box 4163, Berkeley, CA 94704-0163, U.S.A. Prior back issues are obtainable from Periodicals Service Company, 11 Main Street, Germantown, NY 12526-5635. The Pacific Journal of Mathematics is indexed by Mathematical Reviews, Zentralblatt MATH, PASCAL CNRS Index, Referativnyi Zhurnal, Current Mathematical Publications and the Science Citation Index.

The Pacific Journal of Mathematics (ISSN 0030-8730) at the University of California, c/o Department of Mathematics, 969 Evans Hall, Berkeley, CA 94720-3840, is published monthly except July and August. Periodical rate postage paid at Berkeley, CA 94704, and additional mailing offices. POSTMASTER: send address changes to Pacific Journal of Mathematics, P.O. Box 4163, Berkeley, CA 94704-0163.

PJM peer review and production are managed by EditFlow ${ }^{\circledR}$ from Mathematical Sciences Publishers.

\section{PUBLISHED BY}

\section{mathematical sciences publishers}

http://msp.org/

A NON-PROFIT CORPORATION

Typeset in LATEX 


\section{PACIFIC JOURNAL OF MATHEMATICS}

Volume $260 \quad$ No. $2 \quad$ December 2012

\section{Special issue \\ devoted to the memory of Jonathan Rogawski}

In memoriam: Jonathan Rogawski

257

DON BLASIUS, DINAKAR RAMAKRISHNAN and V. S. VARADARAJAN

$p$-adic Rankin $L$-series and rational points on CM elliptic curves

261

Massimo Bertolini, Henri DARMON and KARTIK PRASANNA

The syntomic regulator for $K_{4}$ of curves

AMNON BESSER and ROB DE JEU

Unique functionals and representations of Hecke algebras

381

BENJAMIN BRUBAKER, DANIEL BUMP and SOLOMON FRIEDBERG

A relative trace formula for PGL(2) in the local setting

395

BROOKE FEIGON

On the degrees of matrix coefficients of intertwining operators

433

TOBIAS FINIS, EREZ LAPID and WERNER MÜLlER

Comparison of compact induction with parabolic induction

457

Guy HENNIART and MARIE-FranCE Vigneras

The functional equation and beyond endoscopy

497

P. EDWARD HERMAN

A correction to Conducteur des Représentations du groupe linéaire

HERVÉ JACQUET

Modular $L$-values of cubic level

ANDREW KNIGHTLY and CHARLES LI

On occult period maps

STEPHEN KUDLA and MiCHAEL RAPOPORT

A prologue to "Functoriality and reciprocity", part I

ROBERT LANGLANDS

Truncation of Eisenstein series

EREZ LAPID and KeITH OUELLETTE

Some comments on Weyl's complete reducibility theorem

JONATHAN ROGAWSKI and V. S. VARADARAJAN

On equality of arithmetic and analytic factors through local Langlands correspondence

FREYDOON SHAHIDI 